\title{
Making enactivism even more embodied
}

\author{
Shaun Gallagher \\ Department of Philosophy, University of Memphis; \\ School of Humanities, University of Hertfordshire; \\ Matthew Bower \\ Department of Philosophy \\ School of Humanities and Social Inquiry, University of \\ University of Central Florida \\ Wollongong \\ *s.gallagher[]memphis.edu \\ Received January 2014; accepted August 2014; published Autumn 2014.
}

\begin{abstract}
The full scope of enactivist approaches to cognition includes not only a focus on sensory-motor contingencies and physical affordances for action, but also an emphasis on affective factors of embodiment and intersubjective affordances for social interaction. This strong conception of embodied cognition calls for a new way to think about the role of the brain in the larger system of brain-body-environment. We ask whether recent work on predictive coding offers a way to think about brain function in an enactive system, and we suggest that a positive answer is possible if we interpret predictive coding in a more enactive way, i.e., as involved in the organism's dynamic adjustments to its environment.
\end{abstract}

Keywords: enactivism; sensory-motor contingencies; affect; intersubjectivity; predictive coding.

Enactivism is one version of recently developed embodied approaches to cognition. It offers an approach that is more informed by phenomenology and pragmatism than other versions of embodied cognition, such as the extended mind hypothesis (Clark 2008; Clark and Chalmers 1998), and more radical than the kind of "moderate" (Goldman 2012) or "weak" (Alsmith and Vignemont 2012) embodied cognition found in theorists who locate the body "in the brain" or who translate all bodily action into body-formatted representations (e.g., Berlucci and Aglioti 2010; Gallese and Sinigaglia 2012; Goldman and Vignemont 2010). Enactivists have entered into various debates with these other approaches (see Di Paulo 2009; Gallagher 2011a; Thompson 2007), and clear lines have been drawn to distinguish the differences that involve questions about functionalism, the importance of the body, the way one is coupled to the environment, and so forth. At the same time, however, there are a number of differences to be found within the enactive camp itself, so 
that the "early" enactivism of Varela, Thompson and Rosch (1991), closely continued by De Jaegher and Di Paulo (2007) is not exactly the same as the "middle' enactivism of O'Regan and Noë (2001; Noë 2004), or the "latest enactivism" of Hutto and Myin (2013). There are some obvious differences among these authors simply in terms of pedigree: Varela et al. are strongly influenced by phenomenology (as well as Buddhism), O'Regan and Noë by cognitive science, and Hutto and Myin by analytic philosophy of mind. While enactivists should be pleased with these convergences of disciplines and traditions, we're going to suggest that they should also be critical with respect to the question about how embodied enactivism is.

To pose this critical question we want to focus on the work of those enactivists who emphasize the role played by sensorimotor contingencies in perception, especially O'Regan and Noë. While this is clearly an embodied approach to cognition, we want to argue that it is not as richly embodied as it should be. One starts to see this in some recent authors who have distinguished the O'Regan and Noë brand of enactivism as the 'sensorimotor approach' in contrast to the enactive approach (see, e.g., Kyselo and Di Paolo 2013; also Stapleton 2013). The focus on sensorimotor contingencies emphasizes a narrow conception of embodiment in terms of neuro-muscular function. In contrast to this narrow focus, we want to suggest that these versions of sensorimotor enactivism need to be made more embodied. We'll focus on three issues: (1) affectivity; (2) intersubjectivity; and (3) a dynamic attunement theory of brain function. Emphasis on these issues is consistent with the enactivism associated with Varela, Thompson, and Di Paolo, and offers a positive account of enactivism that goes beyond the incisive critique of representationalism offered by Hutto and Myin. We also note that although the sensory-motor contingency approach focused on perception, the enactivist project concerns more than just perception. That is, the project is to develop an account of cognition more generally, including perceptual judgments and higher-order cognitive processes such as deliberation, decision, memory, and so forth.

Our goal is to make clear that these issues are important ones for continuing development of the enactivist approach. Elsewhere we have developed some critical considerations with respect to the first two issues (Bower and Gallagher 2013). We'll briefly rehearse these considerations in the next two sections. We'll then focus on the third, and argue that to have a more embodied enactivism we need a different understanding of how the brain works. Specifically we want to explore recent predictive coding models from an enactivist perspective. In this regard we generalize suggestions about the "interactive brain hypothesis” made by Di Paolo and De Jaegher (2012). 


\section{Affectivity}

An enactive account of cognition and perception integrates a variety of bodily factors into cognitive processes. The body, understood as what phenomenologists call the "lived body," includes the related notion of a "body schema" (Gallagher 2005). The role of the body schema pertains to motor control and precisely the kind of sensory-motor contingencies emphasized by O'Regan and Noë (2001) and Noë (2004); it facilitates interactions with one's surroundings, and it contrasts to the "body image," a term that designates the ways in which the body shows up for consciousness, in certain circumstances, as its intentional referent.

The lived body in its full sense, however, involves more than the sensorimotor body schema and body image. It involves the full ensemble of bodily factors that govern conscious life, but that operate in a pre-noetic fashion, below the level of conscious monitoring and manipulation. Such factors may or may not be accessible to conscious awareness. They include the large realm of affect. This rich affective set of bodily factors is partially constitutive of perception in ways that go beyond sensorimotor contingencies. An account that focuses only on sensorimotor contingencies falls short due to its neglect of the relevance of the affective aspects including proprioceptive and kinaesthetic aspects-factors that should be of high interest since they derive from movement and contribute to one's practical grasp of sensorimotor contingencies. Affective factors, however, also involve a complex motivational dimension that animates body-world interaction (Bower and Gallagher 2013; Stapleton 2013; Colombetti 2013).

Meaningful encounters with the world imply a perceiving agent with some basic motivation to perceptually engage her surroundings. Schemata of sensorimotor contingencies give an agent the how of perception, a tacit knowledge of potential sensorimotor engagements, without giving its why, which depends on latent valences that push or pull in one direction or another for attention and for potential sensory-motor engagement, reflecting, for example, a degree of desirability. "The endogenously originating motivational viscera of the body are just as important to perception as the exogenously oriented sensory-motor elements" (Bower and Gallagher 2013: 111).

Affects are not restricted to the domain of phenomenal consciousness, although they may certainly have an effect on what experience feels like. I may consciously experience the blues, or I may be unaware that my whole demeanor reflects the blues. Affect is deeply embodied even to the extent that affective phenomena may be constrained by the functioning of the circulatory system. For example, heartbeat influences how and whether fear-inducing stimuli (images of fearful faces, in the reported experiments) are processed (Garfinkel et al. 2013). When the heart contracts in its systole phase, fearful stimuli are more easily recognized, and they tend to be perceived as more 
fearful than when presented in its diastole phase. That is, the fact that, rather than brains in vats, we are flesh and blood creatures equipped with beating hearts, explains in part why we have just the sorts of affective states that we do.

Consider particular instances of the affects involved in hunger and fatigue. Somaesthetic factors such as hunger delimit our perception and action possibilities, as well as our cognitive possibilities. William James once noted that an apple appears larger and more invitingly red when one is hungry than when one is satiated. A recent study (Danziger et al. 2011) reinforced the idea that hunger can shape, and perhaps even distort, cognitive judgment processes. The study shows that the rational application of legal reasons does not sufficiently explain the decisions of judges. Whether the judge is hungry or satiated may play an important role.

The percentage of favorable rulings drops gradually from $\approx 65 \%$ to nearly zero within each decision session [e.g., between breakfast and lunch] and returns abruptly to $\approx 65 \%$ after a [food] break. Our findings suggest that judicial rulings can be swayed by extraneous variables that should have no bearing on legal decisions. (Danziger et al. 2011: 1).

In one sense, such affective factors appear "extraneous” only if we try to think of cognition as something that is disembodied, although clearly they may be extraneous to the formal aspects of legal reasoning. In any case, it seems reasonable to think that this embodied affective aspect of hunger has an effect on the jurist's perception of the facts, as well as on the weighing of evidence, and doesn't appear out of nowhere just when the judicial decision is made.

Typically our embodied condition does not reflect a simple, isolated affectrather, there is a cocktail, a mélange of aspects that make up one's affective state. After a day of trekking up a mountain, one's perception may be informed by a combination of hunger, pain, fatigue, troubled respiration, feelings of dirtiness, and the kinaesthetic difficulty involved in climbing. It's likely that that the mountain path looks more different and less challenging than after a good night's sleep, not because of certain objective qualities that belong to the path, but because of my affective state. Such affective aspects color my perception as they more generally constrain my being-in-the-world.

Affective phenomena are pervasively integrated into our perceptual and cognitive experiences (Pessoa 2013; Barrett and Bliss-Moreau 2009), as noted by many enactivists (Colombetti 2007; Ellis 2005; Thompson 2007; Thompson and Stapleton 2009). Shifts of attention may be led in one direction or another by the affective ebb and flow of what we experience. From a phenomenological perspective Husserl (2004) describes such affective states involving tension, resolution, exertion, unease, and satisfaction/dissatisfaction as modulating our perceptual (but not only perceptual) attention. Attention, in this sense, is embodied in a variety of related ways. In visual experience, for example, attend- 
ing to something may involve squinting or opening the eyes widely, it may involve a contortion of the face all the way from the scalp down to a gaping mouth or pursed lips, and so on (Bergson 2001: 27-28).

Affect is closely related to the phenomenon of perceptual presence elaborated by Noë (2004), and specifically involves a sense of interest or investment. Perceptual presence is the sense one has of the perceptual accessibility of nonapparent aspects or sides of a perceived object, or, more broadly, of what is not directly sensed in the present moment (e.g., the side of the object that is not visible). The notion of "perceptual interest" (Bower and Gallagher 2013) denotes the affective sense of the stakes or the costs involved in exchanges with one's environment. This is not the same as Husserl's concept of the "I can," which signifies the intuitive possession of a sense of skill or competence. Rather, even if one is capable of accomplishing some feat in those terms, one might still not feel "up to the task," or not feel inclined to do the work it might take, which is the affective nuance the sense of interest is supposed to highlight. Thus, interwoven with that perceptual sense of presence is a sense of the affective stakes of making something available or present. To make something available involves definite costs in following through on transactions with environmental affordances. One's environment affords many possibilities for action, but each has its affective price tag, and they are not all equally affordable. One thus not only has a practical (sensorimotor) understanding of accessibility, but an affective take on that same accessibility, in terms of interest or inclination to follow through. The latter may also involve a perceptual sense of the ease or difficulty of making something present.

It's true that a perceiving agent's perceptual stance is determined by a mastery of sensorimotor contingencies needed to access environmental affordances in suitable ways. Such mastery, however, once acquired, may be a relative constant, and more or less generic or standard set of skills suitable for most transactions with the world. Anyone with that same skill set might perceive in the same way. In contrast, the particularities of affect will differ from one individual to another, or from one day to another. Taking affective phenomena into account importantly enriches one's understanding of perception, since it clarifies the nature of individual perspective in perception. A broad spectrum of individual life circumstances may, in terms of affect, be brought to bear on perception, as well as other forms of cognition. These circumstances include not only physical burdens and impediments, such as the impediment of fatigue from physical exertion, but also broader circumstances having to do with time of day, since one typically is energized at the start of the day and tired out toward the end, or with longer-term life phases, since youth and old age surely shape one's perceptual interest. 
While everyone is affected by such circumstances in one way or another, each individual lives them out in a unique way. The sense of the presence of the other side of an object, of what is behind one, of what is in an adjoining room, and the like, touches-over and above one's generic strategies for bodily coping with the environment-one's individual condition with all of its strengths and weaknesses. In a very simple example, what Noë calls the 'grabbiness" of an object is dependent not only on one's sense of the pertinent sensorimotor contingencies, whether the object is near or far, and properly shaped and weighted, etc., and not only on whether one is in a state of pain, or fatigue, or fear, etc., but on whether one is even concerned about (or inclined to) the possibility of grabbing the object.

\section{Intersubjectivity}

A series of experiments conducted by Proffitt et al. (1995; Proffitt 2003) purportedly show that the estimation of distance is influenced by anticipated effort. Subjects saddled with a heavy backpack tend to overestimate perceived distance, whereas those without backpacks do not. Proffitt et al. (1995) similarly describe how subjects overestimate the degree of incline of a slope when fatigued, and this may translate into the subject's lack of inclination (to climb), which further informs perception. The hill looks not only steeper, but also uninviting. This might count as at least indirect evidence that perceptual experience and/or judgment is informed by one's present affective state. These results, however, have been challenged by Durgin et al. (2009), in a way that nicely points in a different, but equally important direction for our considerations here. They show that steeper estimates of incline while wearing a backpack "are judgmental biases that result from the social, not physical, demands of the experimental context” (p. 1). Without awareness of this bias, subjects who sense the aim of the experiment estimate a steeper incline than subjects who are misled about the purpose of the backpack (e.g., that it contains electromyographic equipment to measure muscle tension). In other words, those subjects who had a sense of the experimenters' intentions were biased in favor of those intentions, without necessarily knowing it.

If Proffitt is right (see Proffitt 2009; 2013 for further discussion), his experimental results could be taken to point to the embodied-affective nature of perception. But if Durgin et al. (2009; 2012) are right, their results still point to an embodied phenomenon-namely, the significance of others on our perceptions and/or judgments. ${ }^{53}$

\footnotetext{
${ }^{53}$ We note that there is continuing debate about whether these are effects on perception itself or on perceptual judgments, a distinction that can be clearly made in the experimental lab (see Firestone and Scholl 2014 experiments on the El Greco fallacy). This is an important issue in regard to claims about cognitive penetration of perception, but for our limited purposes here it is sufficient
} 
There are several ways to understand intersubjectivity as an embodied phenomenon. For example, some simulation theorists, like Gallese (2001; Gallese and Sinigaglia 2012) understand empathic consciousness or social cognition to be embodied in at least a weak sense. That is, they understand social cognition to depend on body-formatted neural processes, specifically the activation of mirror neurons (MNs) ${ }^{54}$ A more enactive approach, however, interprets the mirror system to be activated in preparation for or anticipation of a response to the other. That is, MN activation does not ordinarily involve matching (in one's own system) or imitating the action of the other person (see Catmur et al. (2007); Dinstein et al. 2008; Csibra 2005 for empirical evidence; also Gallagher 2008); it involves anticipatory processes that are keyed in with affordances for further interaction, or preparation for a complementary action in response to an observed action (Newman-Norlund et al. 2007). In other words, it is part of a response to social affordances.

On the enactive view, social cognition is characterized by, and sometimes constituted by embodied interaction (Di Jaegher, Di Paulo and Gallagher 2010). This view is usually worked out in contrast to theory of mind (ToM) approaches that emphasize mindreading by either theoretical inference or simulation (Gallagher 2001; 2005; 2008). Intersubjective interaction is not about mindreading the mental states of others, but about directly perceiving their intentions and emotions in their postures, movements, gestures, facial expressions, vocal intonations, etc., as well as in their highly contextualized (by physical environment, social roles, culture, etc.) actions (Gallagher and Varga 2013).

One important aspect of intersubjective interaction that shows up even in cases where we are not explicitly interacting with the other, although another person is present, is the effect that his or her presence has on learning and perception. One can understand this developmentally in regard to how we learn what is important. Studies of "natural pedagogy," where there is explicit interaction, show that how the caregiver relates to the child influences what the child learns. Natural pedagogy, which involves ostensively directing the infant's attention to some object or event,

enables fast and efficient social learning of cognitively opaque cultural knowledge that would be hard to acquire relying on purely observational learning mechanisms alone.... [H] uman infants are prepared to be at the receptive side of natural pedagogy (i) by being sensitive to ostensive signals that indicate that they are being addressed by communication, (ii) by developing referential

that there are such effects on cognition, whether perception, perceptual judgment, memory, etc. and specifically in everyday pragmatic environments.

${ }^{54}$ For the notion of body-formatted (or B-formatted) representations, see Goldman and de Vignemont (2010), and Goldman (2012) who propose a "moderate" or "weak" (Alsmith and de Vignemont 2013) conception of embodied cognition. 
expectations in ostensive contexts and (iii) by being biased to interpret ostensivereferential communication as conveying information that is kind-relevant and generalizable. (Csibra and Gergely 2009: 148).

More generally, it is through our interactions with others that we learn what objects are significant or valuable. We learn to understand the world along these lines of significance and value, and often objects that fall outside of such lines don't even register. In the same way that expert training hones the perceptual system so that experts are able to perceive things that non-experts fail to perceive, in some sense, we all become experts in everyday life through our interactions with others.

Indeed, this intersubjective education of perception and judgment continues throughout life. Adult subjects presented with a face looking towards (or away from) an object evaluate the object as more (or less) likeable than those objects that don't receive much attention from others. If one adds an emotional expression to the face and one get's a stronger effect (Bayliss et al. 2006; 2007). Furthermore, seeing another person act with ease (or without ease) toward an object will influence observers' feelings about the object (Hayes et al. 2007).

Social interactions, social roles and groupings also have their influence on how one perceives the world. In a modification of Proffitt's scenario, imagine being exhausted, but the incline is a hill that you are climbing with friends or to meet a loved one (Schnall et al 2008). Or, again, think of the affective import in situations where one would be seen by others as not up to the task, negatively impacting one's image. In some social circumstances one may find a particular setting to be of more interest and more attention grabbing than if one were with a different group, or alone.

\section{The embodied and enactive brain}

Following the weak embodiment strategy, one might argue that all such effects of affectivity and intersubjectivity are ultimately processed in the brain, so that even the most enactive aspects of perception are reducible to brain processes. After all, even if one interprets MNs as part of an enactive system, they are neurons located in certain brain areas. Moreover, when we look at how the brain works, we need concepts like representation and inference to explain it, and these go against strong enactivist claims that tend to be antirepresentationalist and dismissive of the idea of subpersonal inferences. On the weak embodiment view (e.g., Goldman 2012), claims about enactive perception, affectivity, and intersubjectivity can all fit neatly into orthodox internalist accounts. 
The notion of an enactive system requires conceiving of the brain in a different way. In evolutionary terms, the brain does what it does and is the way it is, across some scale of variations, because it is part of a living body that has hands that can reach and grasp in certain limited ways, and because it has eyes structured to focus, and so on. The sensorimotor system is the way it is because of the kind of organism the human body is. In addition the organism has an autonomic and peripheral nervous systems, and not just a central system. It attains an upright posture, which, in evolutionary terms reshapes essential features, including the brain (Gallagher 2005), allowing the person to cope with specific kinds of environments, and with other people. Changes to any of the bodily, environmental, or intersubjective conditions elicit responses from the organism as a whole. On this view, rather than representing or computing information, the brain is better conceived as participating in the action.

The enactive interpretation is not simply a reinterpretation of what happens extra-neurally, out in the intersubjective world of action where we anticipate and respond to social affordances. An enactive interpretation of the MN system, for example, points beyond the orthodox explanation of information processing to the possibility of rethinking not just the neural correlates of perception or intersubjectivity, but the very notion of neural correlate, and how the brain itself works. More than this, it suggests a different way of conceiving brain function, specifically in nonrepresentational, integrative and dynamical terms (see, e.g., Gallagher et al. 2013; Hutto \& Myin 2013).

This doesn't mean that we should simply turn the issue over to neuroscientists to adjudicate, or simply consult what the neuroscientists say about subpersonal neural processes. Most neuroscientists are Helmholtzian and would endorse the idea that the neural processes underlying perception are inferential and representational. ${ }^{55}$ Even the Bayesian predictive coding account treats perception and object recognition as an inferential process. As Friston puts it, predictive coding is "now a widely accepted view of perception that can be traced back to Helmholtz's original writings on unconscious inference" (2012: 248; also see Clark 2013). ${ }^{56}$

It's obviously important to understand brain dynamics. More generally, we've known for a long time that anticipatory processes are hugely important for perception and action. In neuroscience we have the work of Berthoz (2000),

\footnotetext{
${ }^{55}$ The Helmholtzian idea that perception involves subpersonal inferences may or may not be correct (see Bennett and Hacker 2003; Orlandi 2012; Hutto and Myin 2013).

${ }^{56}$ The unconscious processes performed by the brain "are like inferences insofar as from the observed effect on our senses we arrive at some conception of the cause of this effect. This is the case even though we only in fact have direct access to the events at the nerves; we sense the effects and never the external objects” (Helmholtz 1867: 430).
} 
for example; and in phenomenology, Husserl's account of temporal structure remains important (1991; Gallagher 1998; 2011b). Work in predictive coding provides an account of how neural processing participates in these pervasive dynamic anticipatory processes. But again there are questions of how to interpret what we are learning about such processes. The assumption about visual perception, for example, is that the brain has no direct access to the outside world, so it needs to interpret or decode neuronal firing patterns that are generated by light hitting our retinas. Sensory data "is all the brain has access to" (Hohwy 2013, 13). If you think of this in terms of inference then the brain is seemingly deducing to the best explanation of what has caused a particular pattern of neuronal activation and thereby representing that cause via a process of causal inference. Since a given pattern could be caused by any number of different stimulus configurations, the task involves figuring the probabilities based on current neural states of the system that may relate, for instance, to context.

How does this Bayesian process work in strict neuronal terms? There is general agreement that the process is a hierarchical one involving synaptic inhibition based on an empirical prior-something that depends on the organism's previous experience and context-sensitive learning. This means that specific neural networks, currently in a particular state because the organism has previously encountered a particular stimulus or environment, or has a particular history, determine ongoing processes that lead to top-down synaptic inhibition (a modulation of connections) anticipating further processing consistent with prior processing. Such inhibitory patterns constitute a prediction which is then matched against ongoing sensory input. If there is a mismatch, i.e., if the new stimulus generates a different firing pattern than the one anticipated, prediction errors are sent back up the line and the system adjusts dynamically back and forth until there is a relatively good fit. So on the predictive coding model, this or something like this is going on when we perceive the world.

If that's the case, if these are the kinds of things that are happening in the brain, it's not clear that we need to think of it as a kind of inference rather than a kind of dynamic adjustment process in which the brain, as part of and along with the larger organism, settles into the right kind of attunement with the environment-an environment that is physical but also social and cultural (Bruineberg and Rietveld 2014).

Whether or not we should think that beliefs, values, as well as affective states and perspectives can shape the way that one quite literally sees the world, or can enter into subpersonal processes in terms of predictive coding models (Stapleton 2013), it remains an open question about how the neural (synapticinhibitory) processes described by such models are best characterizedwhether as inferential (e.g., Hohwy 2013), or as part of a dynamical attune- 
ment of organism to environment, and/or in terms of plasticity where sensory-motor neurons have become attuned by associative processes and prior experience. In any case, on the enactive view, the explanatory unit of perception (or cognition, or action, etc.) is not the brain, or even two (or more) brains in the case of social cognition, but a dynamic relation between organisms, which include brains, but also their own structural embodied features that enable specific perception-action loops involving social and physical environments, which in turn effect statistical regularities that shape the structure and function of the nervous system.

The question is, what do brains do as part of a dynamical attunement of organism to environment in the complex mix of transactions that involve moving, gesturing, and interacting with the expressive bodies of others, with their eyes and faces and hands and voices; bodies that are gendered and raced, and dressed to attract, or to work or play; bodies that incorporate artifacts, tools, and technologies, that are situated in various physical environments, and defined by diverse social roles and institutional practices?

Di Paolo and De Jaegher (2012) have proposed the Interactive Brain Hypothesis in order to help model the possible relations between social interaction and neural processes. "The hypothesis states that interactive experience and skills play enabling roles in both the development and current function of social brain mechanisms, even in cases where social understanding happens in the absence of immediate interaction" (p. 1). We can extend this idea beyond the intersubjective context by following the suggestion that the brain is primarily and more generally an organ of relation (Fuchs 2011). Evan Thompson (2014) provides a good analogy to indicate that the mind is relational, and that the brain plays its part in that relationality. Saying that cognition is in the brain is like saying that flight is inside the wings of a bird. But just as flight doesn't exist if there is just a wing, without the rest of the bird, and without an atmosphere to support the process, and without the precise mode of organism-environment coupling to make it possible, so cognition doesn't exist if there is just a brain without bodily and worldly factors. Can this way of thinking be made consistent with predictive coding models?

Barrett and Bar's affective prediction hypothesis suggests a positive answer. On their predictive coding model "responses signaling an object's salience, relevance or value do not occur as a separate step after the object is identified. Instead, affective responses support vision from the very moment that visual stimulation begins" (Barrett and Bar, 2009, p. 1325). At the earliest point of visual processing, the medial orbital frontal cortex is also activated initiating a train of muscular and hormonal changes throughout the body, "interoceptive sensations" from organs, muscles, and joints associated with prior experience, and integrated with current exteroceptive sensory information that helps to guide response and subsequent actions. In this respect, perceiving the 
environment involves not just a set of neurons firing in the brain, but also undergoing certain bodily affective changes that accompany this integrated processing. Before we consciously recognize an object or other person, for what it or he or she is, our bodies are already configured into overall peripheral and autonomic patterns based on prior associations. In terms of the predictive coding model used by Barrett and Bar, priors, that include affect, are not just in the brain, but involve a whole body adjustment to the environmental stimulus.

This suggests that the brain participates in a system, along with eyes and face and hands and voice, and so on. And the brain would work differently if its embodiment lacked eyes, face, hands, voice, and so on. This is a fully embodied system that enactively anticipates and responds to its environment. How an agent responds and what an agent perceives will depend to a great degree on the overall dynamical state of the brain, but also on environmental factors, embodied affective and intersubjective factors, the person(s) with whom she is interacting, her worldly and intentional circumstances, the bodily skills and habits she has formed, her physical condition, as well as her history of personal experiences, and what the other person may expect in terms of normative standards stemming from communal and institutional practices (Gallagher et al. 2013). Change any of these things and we can expect changes in neural processing, not because the brain represents such changes and responds to them in central command mode, but because the brain is part of the larger embodied system that is coping with its changing environment.

\section{Conclusion}

We've argued that it's not enough to model an enactivist approach to perception and cognition on sensorimotor contingencies alone, even if they do play an important role in such matters. There is good evidence that affective and intersubjective aspects of embodiment are also important contributories to perceptual and cognitive processes. In addition, this realization pushes us to re-think the role played by neuronal processes in the brain. Even a neuroscience that frames brain function in terms of predictive coding needs to recognize that the brain is part of a system that attunes to and responds to its environment in a way that enacts a meaning relative to the particularities of its embodiment. 


\section{References}

Alsmith, A. J. T. and De Vignemont, F. 2012. Embodying the mind and representing the body. Review of Philosophy and Psychology. 3 (1): 1-13.

Barrett, L. F. and Bar, M. 2009. See it with feeling: affective predictions during object perception. Philosophical Transactions of the Royal Society of London. Series B, Biological Sciences, 364 (1521):1325-1334.

Barrett, L. F. and Bliss-Moreau, E. 2009. Affect as a psychological primitive. Advances in Experimental Social Psychology 41, pp. 167-218.

Bayliss, A.P., Paul, M.A., Cannon, P.R. \& Tipper, S.P. 2006. Gaze cueing and affective judgments of objects: I like what you look at. Psychonomic Bulletin \& Review 13: 1061-6.

Bayliss, A.P., Frischen, A., Fenske,M.J. \& Tipper, S.P. 2007. Affective evaluations of objects are influenced by observed gaze direction and emotional expression. Cognition 104: 644-53.

Bennett, M.R. and Hacker, P.M.S. 2003. Philosophical Foundations of Neuroscience. Oxford: Blackwell Publishing

Bergson, H. 2001. Time and Free Will. F. L. Pogson (Trans.), Mineola, NY: Dover Publications.

Berlucchi, G. and Aglioti, S. 2010. The body in the brain revisited. Exp. Brain Res. 200: 25-35.

Berthoz, A. 2000. The Brain's Sense of Movement. Cambridge: Harvard University Press.

Bower, M. and Gallagher, S. 2013. Bodily affectivity: Prenoetic elements in enactive perception. Phenomenology and Mind 2:108-131.

Bruineberg, J. and Rietveld, E. 2014. Self-organization, free energy minimization, and optimal grip on a field of affordances. Frontiers in Human Neuroscience. 8: 1-14

Catmur, C., V. Walsh, and C. Heyes. 2007. Sensorimotor learning configures the human mirror system. Current Biology 17(17): 1527-1531.

Clark, A. 2008. Supersizing the Mind: Reflections on Embodiment, Action, and Cognitive Extension. Oxford: Oxford University Press.

Clark, A. 2013. Whatever next? Predictive brains, situated agents, and the future of cognitive science. Behavioral and Brain Sciences 36(3), pp. 181-204.

Clark, A. And Chalmers, D. 1998. The extended mind. Analysis 58 (1): 7-19.

Colombetti, G. 2007. Enactive appraisal. Phenomenology and the Cognitive Sciences 6: 527-546.

Colombetti, G. 2013. The Feeling Body: Affective Science Meets the Enactive Mind, Cambridge, MA: MIT Press.

Csibra, G. 2005. Mirror neurons and action observation. Is simulation involved? ESF Interdisciplines. http://www.interdisciplines.org/mirror/papers/. 
Csibra, G., and G. Gergely. 2009. Natural pedagogy. Trends in Cognitive Sciences 13: 148-153.

Danziger, S., J. Levav, L. Avnaim-Pesso. 2011. Extraneous factors in judicial decisions. PNA S.First published online: www.pnas.org/cgi/doi/10.1073/pnas.

De Jaegher, H., and Di Paolo, E. 2007. Participatory Sense-Making: An enactive approach to social cognition. Phenomenology and the Cognitive Sciences 6, 485-507

De Jaegher, H., Di Paolo, E. and Gallagher, S. 2010. Does social interaction constitute social cognition? Trends in Cognitive Sciences 14 (10): 441-447.

Dinstein, I., C. Thomas, M. Behrmann, and D.J. Heeger. 2008. A mirror up to nature. Current Biology 18 (1): R13-R18.

Di Paolo, E. 2009. Extended life. Topoi 28 (1): 9-21.

Di Paolo, E., \& De Jaegher, H. 2012. The interactive brain hypothesis. Frontiers in human neuroscience, 6 .

Durgin, F.H., Baird, J.A., Greenburg, M., Russell, R., Shaughnessy, K., \& Waymouth, S. 2009. Who is being deceived? The experimental demands of wearing a backpack. Psychonomic Bulletin \& Review 16: 964-969.

Durgin, F. H., Klein, B., Spiegel, A., Strawser, C. J., \& Williams, M. 2012. The social psychology of perception experiments: Hills, backpacks, glucose, and the problem of generalizability. Journal of Experimental Psychology: Human Perception and Performance, 38(6), 1582.

Ellis, R. 2005. Curious Emotions: Roots of Consciousness and Personality in Motivated Action. Philadelphia: John Benjamins Publishing Company.

Firestone, C., \& Scholl, B. J. 2014. "Top-down” effects where none should be found the El Greco Fallacy in perception research. Psychological Science 25(1): 38-46.

Friston, K. 2012. Prediction, perception and agency. International Journal of Psychophysiology 83 (2): 248-252.

Fuchs T. 2011. The brain: a mediating organ. J. Conscious. Stud. 18: 196-221

Gallagher, S. 1998. The Inordinance of Time. Evanston: Northwestern University Press.

Gallagher, S. 2001. The practice of mind: Theory, simulation, or primary interaction? Journal of Consciousness Studies, 8 (5-7): 83-107

Gallagher, S. 2005. How the Body Shapes the Mind. Oxford: Oxford University Press.

Gallagher, S. 2008. Inference or interaction: Social cognition without precursors. Philosophical Explorations 11 (3): 163-73.

Gallagher, S. 2011a. Interpretations of embodied cognition. In W. Tschacher and C. Bergomi, eds. The Implications of Embodiment: Cognition and Communication (59-71) Exeter: Imprint Academic.

Gallagher, S. 2011b. Time in action. Oxford Handbook on Time (419-37). C. Callender, ed. Oxford: Oxford University Press.

Gallagher, S. 2007. Simulation trouble. Social Neuroscience. 2 (3-4), 353-65. 
Gallagher, S. and Varga, S. 2013. Social constraints on the direct perception of emotions and intentions. Topoi. DOI 10.1007/s11245-013-9203-x

Gallagher, S., Hutto, D. D. Slaby, J. and Cole, J. 2013. The brain as part of an enactive system. Behavioral and Brain Sciences 36 (4): 421-422.

Gallese V. 2001. The 'shared manifold' hypothesis: From mirror neurons to empathy. Journal of Consciousness Studies 8: 33-50.

Gallese, V., \& Sinigaglia, C. 2011. What is so special about embodied simulation? Trends in cognitive sciences, 15(11), 512-519.

Garfinkel, S., Minati, L. and Critchley, H. 2013. Fear in your heart: Cardiac modulation of fear perception and fear intensity. Poster presented at the British Neuroscience Association Festival of Neuroscience. (8 April 2013).

Goldman, A. and De Vignemont, F. 2009. Is social cognition embodied? Trends in Cognitive Sciences 13 (4), 154-59.

Hayes, A. E., Paul, M. A., Beuger, B., \& Tipper, S. P. 2008. Self produced and observed actions influence emotion: The roles of action fluency and eye gaze. Psychological research, 72(4), 461-472.

Helmholtz, H. 1867. Handbuch der Physiologishen Optik. Leipzig: Leopold Voss.

Hohwy, J. 2013. The Predictive Mind. Oxford: Oxford University Press.

Husserl, E. 2004. Wahrnehmung und Aufmerksamkeit: Texte aus dem Nachlass (18931912). T. Vongehr and R. Giuliani, eds. Dordrecht: Springer.

Husserl, E. 1991. On the Phenomenology of the Consciousness of Internal Time (19831917). Trans. J. Brough. Dordrecht: Kluwer Academic Publishers.

Hutto, D. and E. Myin 2013. Radicalizing Enactivism: Basic Minds Without Content. Cambridge, MA: MIT Press.

Kyselo, M. and Di Paolo, E. (in press). Locked-in syndrome: A challenge for embodied cognitive science. Phenomenology and the Cognitive Sciences.

Newman-Norlund, R.D., M.L. Noordzij, R.G.J. Meulenbroek, and H. Bekkering. 2007. Exploring the brain basis of joint attention: Co-ordination of actions, goals and intentions. Social Neuroscience 2(1): 48-65.

Noë, A. 2004. Action in Perception. Cambridge, MA: MIT Press.

O'Regan, K. and Noë, A. 2001. A sensorimotor account of vision and visual consciousness. Behavioral and Brain Sciences 23, 939-973.

Orlandi, N. 2012. Embedded seeing-as: Multi-stable visual perception without interpretation. Philosophical Psychology 25 (4): 555-573.

Pessoa, L. 2013. The Cognitive-Emotional Brain: From Interactions to Integration, Cambridge, MA: MIT Press.

Proffitt D. R. 2009. Affordances matter in geographical slant perception. Psychonomic Bulletin \& Review 16: 970-972. 
Proffitt, D. R. 2013. An embodied approach to perception by what units are visual perceptions scaled?. Perspectives on Psychological Science, 8(4), 474-483.

Proffitt, D., M. Bhalla, R. Gossweiler, and J. Midgett (1995). Perceiving geographical slant. Psychonomic Bulletin \& Review. 2(4). 409-428.

Proffitt, D., J. Stefanucci, T. Banton, and W. Epstein (2003). The role of effort in perceiving distance. Psychological Science 14(2), 106-112.

Schnall, S., Harber, K. D., Stefanucci, J. K., \& Proffitt, D. R. 2008. Social support and the perception of geographical slant. Journal of Experimental Social Psychology 44(5), 1246-1255.

Stapleton, M. 2013. Steps to a 'properly embodied' cognitive science. Cognitive Systems Research 22-23, pp. 1-11.

Thompson, E. 2014. The embodied mind: An interview with Evan Thompson. Fall 2014. http://www.tricycle.com/interview/embodied-mind

Thompson, E. 2007. Mind in Life: Biology, Phenomenology and the Sciences of Mind, Cambridge, MA: Harvard University Press.

Thompson, E. and Stapleton, M. 2009. Making sense of sense-making: Reflections on enactive and extended mind theories. Topoi 28: 23-30.

Varela, F. J., Thompson, E. and Rosch, E. 1991. The Embodied Mind: Cognitive Science and Human Experience. Cambridge: MIT Press. 